\title{
Higher order nuclear organization: Three-dimensional distribution of small nuclear ribonucleoprotein particles
}

\author{
(nuclear structure/RNA processing)
}

\author{
DAVid L. SPECTOR \\ Cold Spring Harbor Laboratory, Cold Spring Harbor, NY 11724 \\ Communicated by James D. Watson, September 15, 1989
}

\begin{abstract}
The structural and functional organization of the cell nucleus has been investigated using three-dimensional reconstruction, immunoelectron microscopy, and high-resolution in situ autoradiography. Nuclear regions enriched in small nuclear ribonucleoprotein particles (snRNPs) form a reticular network within the nucleoplasm that extends between the nucleolar surface and the nuclear envelope. The snRNPs occupy $\approx 18 \%$ of the volume of $C H O C \mathbf{4 0 0}$ cell nuclei. The in situ sites of DNA replication and transcription are complementary to, rather than coincident with, the nuclear regions concentrated in snRNPs. Based on these data a three-dimensional model of the organization of the mammalian cell nucleus is presented.
\end{abstract}

While the cell nucleus serves as the repository of the cell's genome, little information is available about the spatial organization of functional components within this organelle. During the interphase portion of the cell cycle, transcription of genes is turned on and off at precise times, DNA is replicated and pre-messenger ribonucleic acid (pre-mRNA) molecules, coding for specific proteins, are processed and transported through the nucleoplasm to the nuclear pores where they are extruded into the cytoplasm as messenger ribonucleoprotein particles. However, relatively little is known about the precise nuclear locations in which these events take place.

Intracellular spatial organization within biological systems is a central factor influencing function at various levels. A striking example of spatial organization is seen in the syncytial blastoderm stages of Drosophila embryogenesis $(1,2)$. During this stage of development, the Drosophila embryo is a single cell containing hundreds of nuclei. It has been shown that the spatial distribution of nuclei influences their subsequent cellularization and developmental fate. Furthermore, Drosophila polytene chromosomes have been differentially stained with vital dyes and in conjunction with optical sectioning methods it has been shown that these chromosomes are closely associated with the inner surface of the nuclear membrane and contact the membrane at specific sites (3-6). The cytoplasm represents a strong precedent where processes are organized within membrane-bounded compartments such as mitochondria, lysosomes, the Golgi apparatus, and the endoplasmic reticulum. At the cytoplasmic organelle level there is evidence suggesting that cytoplasmic microtubules are involved in determining cell polarity (7), influencing the distribution of other cytoskeletal components (microfilaments, intermediate filaments), and generally in organizing the cytoplasm (8-11). Although the nucleus has no membrane-bounded compartments, it may also be organized into functional domains that have for the most part heretofore been undefinable.

The publication costs of this article were defrayed in part by page charge payment. This article must therefore be hereby marked "advertisement" in accordance with 18 U.S.C. $\$ 1734$ solely to indicate this fact.
A new set of organizational criteria that differentiates functional compartments within the nucleus may be needed. One of the most noteworthy examples of the relationship between spatial organization and cell function is represented by the nucleolus, which is a distinct biochemical and structural entity within which ribosomal genes and their products are sequestered from the rest of the genome and nucleoplasm. Within this highly specialized non-membranebounded region of the nucleus ribosomal gene transcription, ribosomal RNA processing, and preribosomal particle formation occur (12). However, analogous nuclear compartmentalization of events or processes involving transcription by RNA polymerases II or III or processing of their transcripts has not yet been demonstrated. Recently, experimental evidence for a nonrandom distribution of mRNA molecules in the cytoplasm has been presented (13). In the present study I have used specific probes and three-dimensional (3-D) reconstruction techniques to identify subnuclear regions associated with transcription, DNA replication, and pre-mRNA processing. Based on these results I propose a model for the structural and functional organization of the mammalian cell nucleus.

\section{MATERIALS AND METHODS}

Cell Culture. CHOC 400 cells obtained from Nicholas Heintz (University of Vermont College of Medicine) were grown on glass coverslips or in 35-mm-diameter Petri dishes in Dulbecco's modified Eagle's medium (GIBCO)/10\% fetal bovine serum.

Immunofluorescence. Cells were prepared for immunofluorescence microscopy by published procedures (14). AntiSm primary antibody $(14,15)$ was used at a dilution of $1: 250$ and fluorescein isothiocyanate conjugated goat anti-mouse IgG (Cappel Laboratories) was used at a dilution of $1: 30$ for $1 \mathrm{hr}$ at $20^{\circ} \mathrm{C}$.

Immunoelectron Microscopy. Cells were prepared for immunoelectron microscopy by previously published procedures (14). Samples were examined in a Hitachi H-7000 transmission electron microscope operated at 60 or $125 \mathrm{kV}$.

3-D Reconstructions. For 3-D reconstruction cells were incubated with 3- $\mu \mathrm{m}$-diameter lectin-coated (Con A) polystyrene spheres for $16 \mathrm{hr}$, fixed, and prepared for immunoelectron microscopy (14). Serial sections were collected on Formvar-coated slot grids and photographs were taken at 60 $\mathrm{kV}$. Data were analyzed using a 3-D reconstruction program with a Kontron image analysis system (IBAS) (Zeiss). Cells that took up three to six spheres were selected for reconstruction. The centers of the spheres in each section were used to align the serial section micrographs before data were entered into the computer program. Data were interactively entered into the system by contouring structures on photographic prints with the use of a mouse. For each image the

Abbreviations: 3-D, three dimensional; pre-mRNA, pre-messenger ribonucleic acid; snRNP, small nuclear ribonucleoprotein particle. 
nuclear envelope, immunostained regions, and nucleoli were entered into the computer program in separate channels so that is was possible to look at a reconstruction of the nuclear envelope alone, of the immunostained regions, of nucleoli, or of all of these structures combined. By using tilt and rotation parameters images were reconstructed at various angles and viewed as stereo pairs to obtain 3-D information.

In Situ Autoradiography. Cells were incubated with $\left[{ }^{3} \mathrm{H}\right]$ thymidine $(50 \mu \mathrm{Ci} / \mathrm{ml} ; 1 \mathrm{Ci}=37 \mathrm{GBq})$ or $\left[{ }^{3} \mathrm{H}\right]$ uridine $(50$ $\mu \mathrm{Ci} / \mathrm{ml}$ ) in culture medium for 2 min on ice. Then the medium was changed and the cells were incubated in prewarmed medium $\left(37^{\circ} \mathrm{C}\right)$ with the labeled compound for $30 \mathrm{sec}$ at $37^{\circ} \mathrm{C}$. Cells were washed with medium containing unlabeled uridine or thymidine at $1 \mathrm{mg} / \mathrm{ml}$ for two 5 -min periods and fixed for immunolabeling (14). Sections $0.5 \mu \mathrm{m}$ thick were attached to precleaned Formvar-coated glass slides and a monolayer of Ilford L4 emulsion was applied by the dipping method. Slides were stored at $4^{\circ} \mathrm{C}$ and developed after 150 days' exposure by the elon gold latensification method (16). Sections were examined in a Hitachi H-7000 transmission electron microscope operated at $125 \mathrm{kV}$.

\section{RESULTS}

Several major classes (U1, U2, U4/U6, and U5) of small nuclear ribonucleoprotein particles (snRNPs) play a crucial role in the processing of pre-mRNA molecules before the latter are translocated out of the cell nucleus (for review, see refs. 17 and 18). I have been interested in the 3-D distribution of these particles within the cell nucleus and in their spatial organization with regard to the in situ localization of the sites of DNA replication and transcription. CHOC 400 cells immunostained and examined by immunofluorescence microscopy routinely exhibit 20-50 irregularly shaped immunofluorescent speckles within each cell nucleus (Fig. 1a). Nucleoli are not immunolabeled by these antibodies (Fig. 1a). Antibodies that recognize proteins common to all major snRNP particles (anti-Sm) as well as antibodies recognizing only $\mathrm{U} 1$ (anti-RNP) or U2 specific proteins (anti-U2) or the 5'terminal 2,2,7-trimethylguanosine cap $\left(\mathrm{m}_{3} \mathrm{G}\right.$ cap) structure of the snRNAs localize to all of the speckles in a given nucleus $(20,21)$. This immunostaining pattern has been observed in cell nuclei irrespective of the fixative used (formaldehyde, glutaraldehyde, methanol, acetone) and in unfixed cells that have been permeabilized with Triton X-100 (data not shown). This speckled staining pattern was observed in 20 cell or tissue types.

On closer examination by immunoelectron microscopy (Fig. 1b) it appeared that the speckled regions were not isolated islands of immunoreactivity but were connected to each other, possibly forming a network or reticulum within the nucleoplasm. In an earlier study I examined the relationship of DNA to this nuclear reticulum using the fluorochrome Hoechst 33258 and showed that the areas occupied by snRNPs were exclusive of the areas of the nucleoplasm that contained the bulk of DNA (22). The snRNP immunostaining pattern is not sensitive to DNase I digestion. In contrast, upon digestion with RNase A, snRNPs become diffusely distributed throughout the nucleoplasm excluding the nucleoli (22). Since the nuclear speckles are present in cells that have been treated with DNase and in cells that have been permeabilized and immunolabeled without prior fixation, it is unlikely that these speckles represent a soluble pool of nuclear components trapped between areas of DNA. It appears that these particles may be attached to, or may themselves make up, a distinct interphase nuclear region independent of the presence of DNA.

To examine the precise 3-D organization of snRNPs within the constraints of the nuclear envelope, serial section immunoelectron microscopy was performed, and the cell nucleus was reconstructed. Stereo pairs of a 3-D model of a reconstruction from seventeen $100-\mathrm{nm}$ serial sections that span the entire cell nucleus are shown in Fig. 2. The nucleus appears as a flattened structure, in the $z$ axis, since it is from a cell that had grown attached to a flat substrate. The cell is $3.5-4.0 \mu \mathrm{m}$ thick. The distribution of snRNPs appears to extend between the nucleolar surface and the nuclear envelope forming a reticular network. The contact between the snRNP clusters and the nucleolar surface may represent a fortuitous interaction resulting from the nucleolus being a barrier to the extension of snRNP clusters. Alternatively, the nueleolar surface may in some way interact with components sof the snRNP network to anchor these structures within the nucleoplasm.

Several portions of the snRNP network extend for a significant distance in the $z$ axis before connecting to an adjacent cluster to form structures that appear as columns (Fig. $2 a$ and $b$, lower portion of the nucleus). This would explain why in many immunofluorescent images, which are focused in one focal plane, it sometimes appears that the regions of the snRNP network are not connected. The nucleoli (blue regions) appear to lie closer to the upper surface of the nucleus where they attach to the nuclear envelope (red or pink lines). If the cell is tilted in the $y$ axis, distinct connections of the snRNP reticulum to the nuclear envelope are more easily observed (Fig. $2 c$ and $d$ ). The black regions between the pseudocolored structures within the nucleus represent the localization of DNA and other nuclear components. The nuclear volume occupied by snRNPs is equal to $158 \mu \mathrm{m}^{3}(18 \%)$, the nuclear volume occupied by the
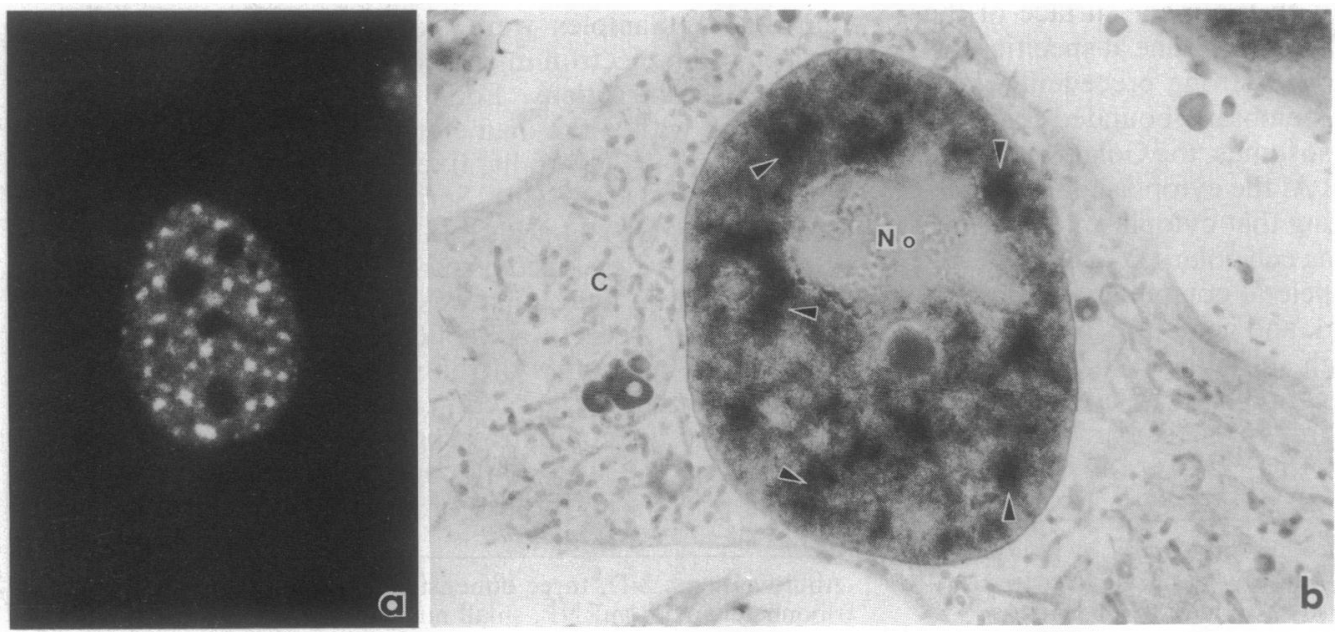

FIG. 1. (a) Immunofluorescence localization of snRNPs using an anti-Sm monoclonal antibody (14). About 20-50 snRNP clusters are present within the cell nucleus. $(\times 1050$. $)(b) \mathrm{Sec}-$ tion ( $0.5 \mu \mathrm{m}$ thick) of a $\mathrm{CHOC}$ 400 cell showing distribution of snRNPs (anti-Sm antibody) by immunoperoxidase staining. Arrowheads point to speckles enriched in snRNPs that appear to connect forming a network. The nucleolus (No) and cytoplasm (C) do not stain. (Section not poststained; $\times 2800$.) 

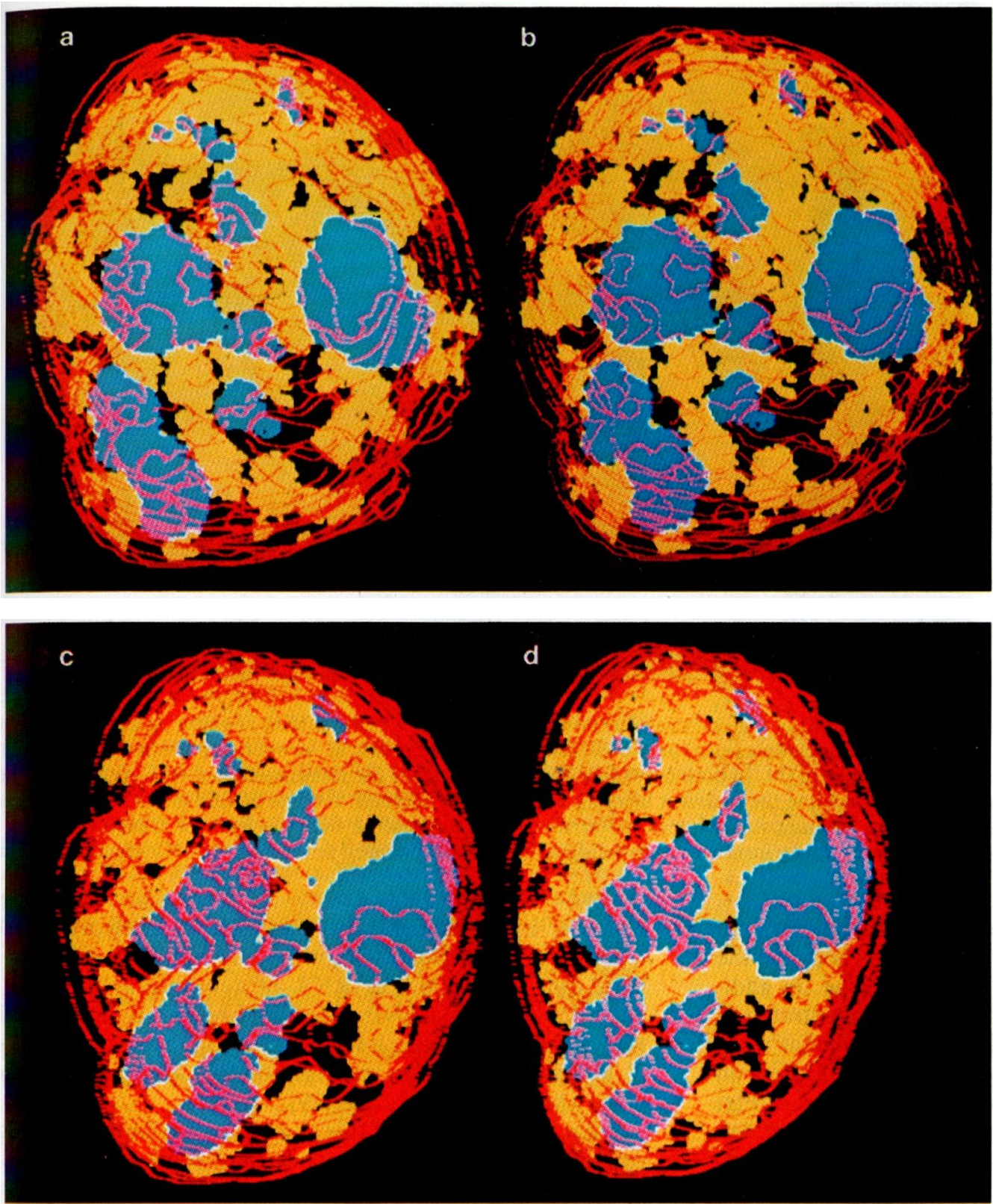

Fig. 2. Model of a 3-D reconstruction of a CHOC 400 cell nucleus showing the distribution of snRNPs (yellow', nucleoli (blue), and the nuclear envelope (red or pink line around each section). ( $a$ and $b$ ) Stereo pair providing a view from the bottom surface of the cell looking up toward the cell surface. ( $c$ and $d$ ) Stereo pair providing a view of the cell tilted in the $y$-axis so that connections of the snRNP clusters to the nuclear envelope are more apparent.

nucleoli is equal to $140 \mu \mathrm{m}^{3}(17 \%)$, and the remaining portions of the nucleoplasm, which contain DNA and other nuclear components, occupy $552 \mu \mathrm{m}^{3}$ (65\%).

I was next interested in identifying where the specific sites of DNA replication and transcription were localized within the cell nucleus in comparison to the regions in which the snRNPs were concentrated. Eukaryotic chromatin has been divided into two classes based on its state of condensation during interphase (23). Heterochromatin is a form that is condensed during interphase and, as such, is generally considered transcriptionally inactive. It is commonly localized in a band around the nuclear periphery and around the nucleolus as well as in patches throughout the nucleoplasm (23). Euchromatin is diffusely distributed throughout the interphase nucleus (23). To identify the sites of DNA replication CHOC 400 cells were labeled with $\left[{ }^{3} \mathrm{H}\right]$ thymidine. Using this procedure to examine 200 cells, I have identified two autoradiographic patterns of DNA replication, a diffuse nucleoplasmic pattern (Fig. $3 a$ ), representing the replication of euchromatin. and a primarily perinuclear and perinucleolar pattern (Fig. 3b), representing the replication of heterochromatin. Both of the autoradiographic patterns observed after $\left[{ }^{3} \mathrm{H}\right]$ thymidine incorporation are complementary to, rather than coincident with, the snRNP-enriched nuclear reticulum supporting the light microscopy studies (22) showing DNA to be absent from these nuclear regions.
Since the transcription and processing of RNA polymerase II transcripts in Drosophila have been proposed to occur simultaneously (24), I was interested in determining the spatial relationship between the sites of transcription and the localization of the snRNP-enriched reticulum within the mammalian cell nucleus. To evaluate the sites of active transcription, and compare them to the snRNP staining region, cells were labeled with $\left[{ }^{3} \mathrm{H}\right]$ uridine for $30 \mathrm{sec}$ prior to fixation and immunolabeling. The short labeling period allows labeling of ribosomal ribonucleic acid and pre-mRNA, which have high turnover rates in the nucleus $\left[t_{1 /,}, 15-180 \mathrm{~min}\right.$ (25)] but of virtually none of the snRNAs, which have a half-life of several cell cycles (26). Similar to that found using $\left[{ }^{3} \mathrm{H}\right]$ thymidine incorporation the $\left[{ }^{3} \mathrm{H}\right]$ uridine label was not coincident with the snRNP-enriched nuclear reticulum (Fig. $3 c)$. The highest concentration of $\left[{ }^{3} \mathrm{H}\right]$ uridine labeling was found over the nucleoli, and lesser amounts of label were distributed within the nucleoplasm. In many instances autoradiographic grains appeared around the snRNP clusters (Fig. 3c, arrows) suggesting that transcription is not occurring within these nuclear regions but may occur around them.

\section{DISCUSSION}

Using 3-D reconstruction techniques combined with immunoelectron microscopy, this study has provided information concerning the in situ 3-D organization of components in- 


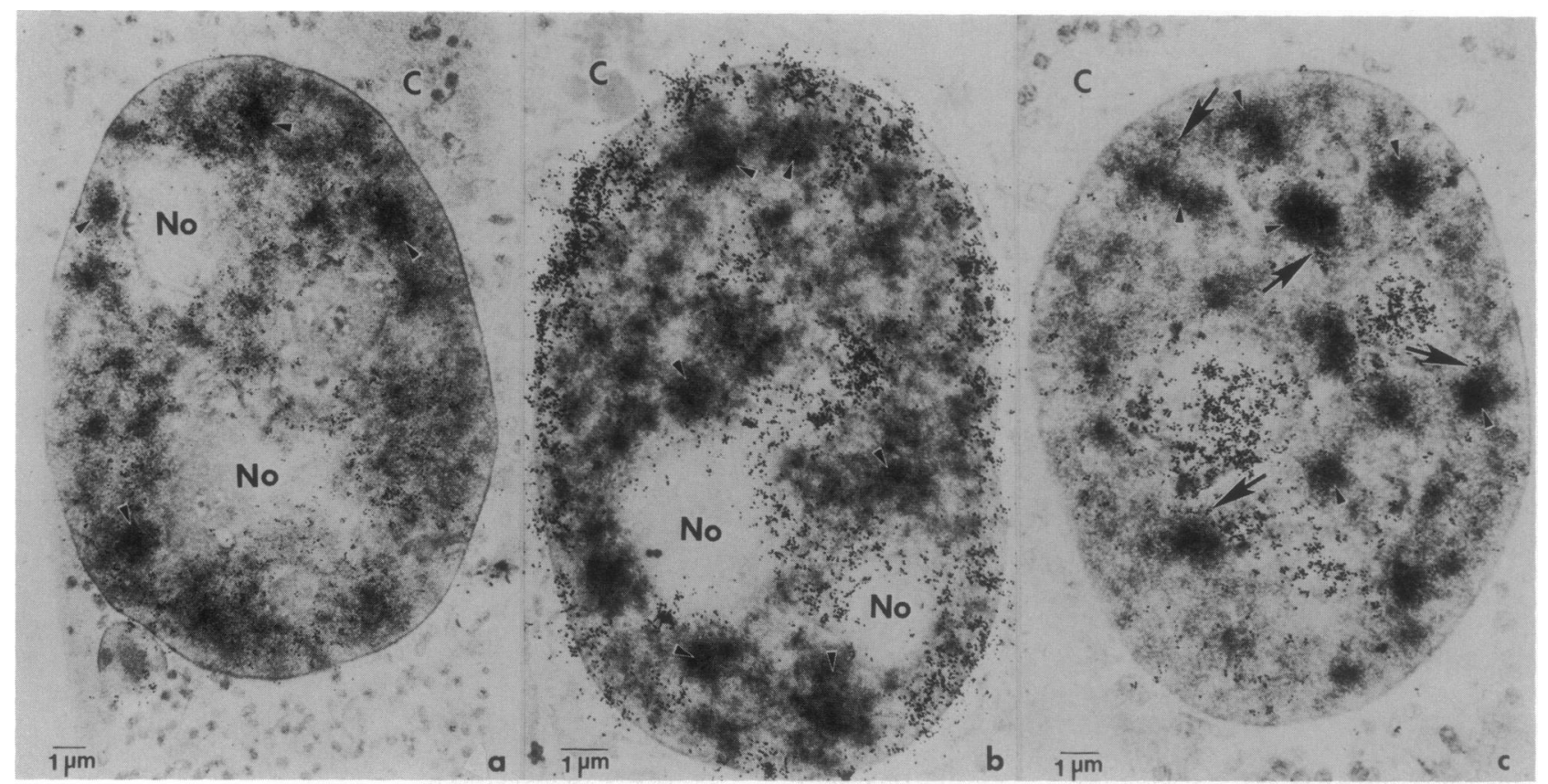

FIG. 3. ( $a$ and $b)$ Cells in the S phase (DNA replication) of the cell cycle at the time of labeling $\left(\left[{ }^{3} \mathrm{H}\right]\right.$ thymidine) exhibit an autoradiographic grain pattern that forms a fine meshwork throughout the nucleoplasm $(a)$ or shows concentrations of grains in the nuclear periphery, perinucleolar regions, and several patches within the central nucleoplasm $(b)$. In both cases few grains appear over the immunostained regions (arrowheads). $(a, \times 3500 ; b, \times 4900$. $)(c)$ In all cells labeled with $\left[{ }^{3} \mathrm{H}\right]$ uridine the heaviest labeling was over the nucleoli, the site of ribosomal RNA synthesis. Cells showed few autoradiographic grains over the immunostained regions (arrowheads), suggesting that the bulk of transcription is not coincident with the snRNP-enriched nuclear regions. In many instances autoradiographic grains appeared around the snRNP clusters (arrows). $(\times 4200$.)

volved in pre-mRNA splicing. I have shown that snRNPs are concentrated in a reticular network that extends between the surface of the nucleolus and the nuclear envelope. In addition, I have shown that the nuclear regions concentrated in snRNPs are complementary to, rather than coincident with, the sites of DNA replication and transcription. Based on these data I propose the following model (illustrated in Fig. 4) for the organization of the mammalian cell nucleus. snRNPs (orange structures) are distributed in a reticular network that extends between the nucleolar surface (blue region) and the nuclear envelope (gold region). The bulk of nuclear DNA is distributed around these regions, but it is not coincident with the snRNP clusters (for example, lavender helices). Transcription of pre-mRNAs takes place adjacent to the snRNP clusters. The transcript may then extend into the snRNP cluster as it is being transcribed (upper right black arrowhead) so that RNA processing and transcription may be concomitant processes. Alternatively, the transcript may be complete before it enters the snRNP cluster (upper left black arrowhead). The timing of transcript entry into the snRNP cluster and/or the interaction of the transcript with the snRNPs may be dependent on factors that may provide a mechanism for posttranscriptional regulation of gene expression. The transcript may move through the snRNP network to the nuclear envelope, during which time the various steps involved in RNA processing would take place. Alternatively, the transcript may take another pathway through the nucleoplasm, which has not yet been defined. After the events of RNA processing are completed, the mature mRNA will leave the nucleus through the nuclear pores (white arrowheads) as a messenger ribonucleoprotein particle (pink structures).

The reticulum concentrated in snRNPs appears to form a "framework" within which events involved in pre-mRNA processing take place or from which they emanate. Since filamentous structures such as actin or tubulin, which appear to be involved in cytoplasmic architecture and organization $(8,27)$, have not as yet been localized within these snRNP clusters, it is unclear how this snRNP network is held together or maintained within the nucleus. One might speculate that RNA-RNA or RNA-protein interactions are involved, since the snRNP reticulum breaks up on incubation with RNase; however, it is resistant to DNase and high salt $(2 \mathrm{M} \mathrm{NaCl})$ extraction and is therefore present in nuclear matrix preparations $(22,28,29)$.

What is the function of this elaborate distribution pattern of snRNPs? The snRNP reticulum may represent processing sites of pre-mRNAs or pathways of pre-mRNAs which are spliced as they are transported through the nucleoplasm to the nuclear envelope. Data to support such a model come from the fact that the network extends to and comes into direct contact with the nuclear envelope-lamina (Fig. 2). A recent study using in situ hybridization to localize Epstein-Barr virus RNA transcripts in Namalwa cell nuclei has shown that transcripts accumulate, forming a bright focus referred to as a "track" within the nucleus (30) that extends toward the nuclear envelope. Lawrence et al. (30) have proposed that the tracks represent RNA transport pathways; however, because of the nature of the studies it is difficult to distinguish whether these tracks actually represent transport pathways or sites of accumulation of viral message since transcripts of the gene examined accumulate in the cell nuclei (31), and essentially no RNA was detected in the cytoplasm. It is possible that the tracks represent a portion of the snRNP-enriched nuclear reticulum described in this paper. Data presented here showing that the sites of transcription are not coincident with the snRNP. enriched nuclear reticulum raise the possibility that transcripts may move from the site of transcription, adjacent to the snRNP clusters, to the nuclear regions enriched in snRNPs (Fig. 4, upper left black arrowhead) where they are processed. This opens the possibility that factors may regulate or coordinate the movement of pre-mRNAs into the snRNP cluster and suggests an unusual mechanism of posttranscriptional regulation of gene expression. Alternatively, the transcript may loop into the snRNP-enriched nuclear region as it is being 


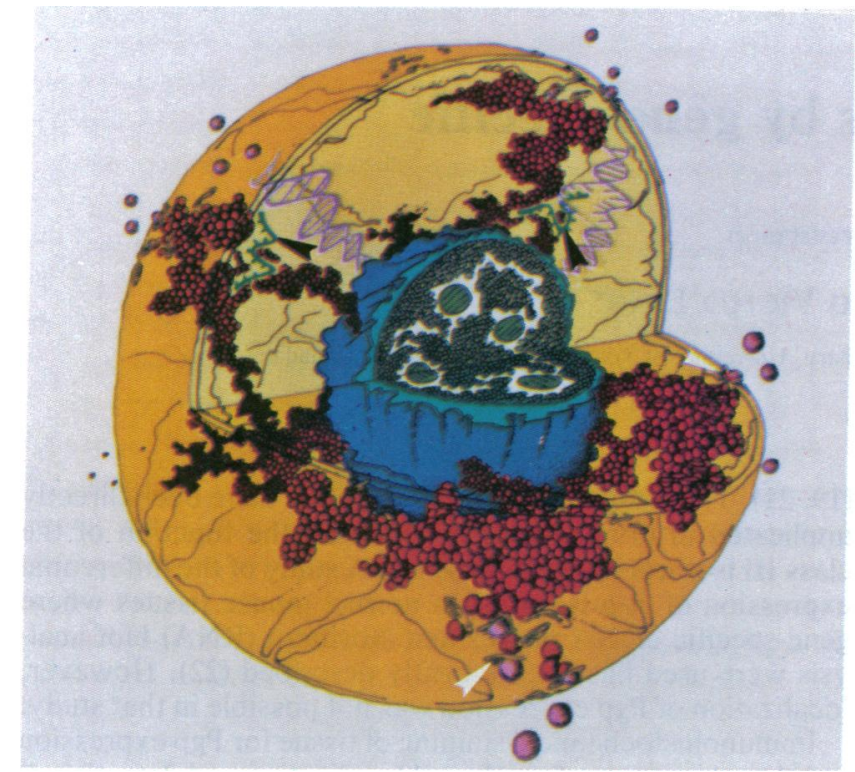

Fig. 4. Model of the 3-D organization of the mammalian cell nucleus based on data presented here. snRNPs (orange structures) are distributed in a reticular network extending between the nucleolar surface (blue region) and the nuclear envelope (gold region). Fibrillar centers (green circles), fibrillar regions (white area), and granular regions containing pre-ribosomal particles (green granules) are observed within the nucleolus (blue region). The bulk of nuclear DNA is distributed around the snRNP-enriched reticulum but is not coincident with the snRNP clusters. Transcripts (black arrowheads) may move through the snRNP network to the nuclear envelope or, alternatively, they may take an as yet undefined pathway through the nucleoplasm. After the events of RNA processing are completed, the mature mRNA will leave the nucleus through the nuclear pores (white arrowheads) as a messenger RNP particle (pink structures).

transcribed (Fig. 4, upper right black arrowhead). This second possibility would be consistent with the data of Beyer $e t$ al. (24) and of Beyer and Osheim (32) for Drosophila where it has been proposed that transcription and RNA processing are concomitant processes. It is also possible that the snRNP. enriched nuclear region may represent a storage area for snRNPs, which may leave these areas in immunologically undetectable numbers and move to the sites of transcription and/or RNA processing.

In a review of mRNA transport Webb et al. (33) suggested that the RNP network observed in nuclear matrix preparations may serve as an assembly line or conveyor belt from the gene to the vicinity of the nuclear pore and furthermore suggested the possibility of related genes or gene families directing their products (mRNAs) to specific nuclear pores. In another hypothesis, nuclear pore complexes have been proposed to function as "gene gating" organelles that contribute to the organization of the 3-D structure of the genome (34). This hypothesis suggests that nuclear pore complexes serve as organelles, each of which interacts with one or more specific genes, and transcripts from a gene will exit the nucleus via the nuclear pore complex to which its gene is gated. No data presently exist to support this hypothesis; however, my findings that nuclear components involved in pre-mRNA processing extend out to the nuclear periphery are certainly compatible with such a model. It is possible that nuclear pores are concentrated at the sites where the snRNP network interacts with the nuclear envelope-lamina. In addition, since DNA in the nucleus is distributed around areas that are enriched in snRNPs, another possibility is the idea that genes located adjacent to a particular region of the snRNP network will have their pre-mRNAs processed at this region of the snRNP network. This idea is particularly exciting in light of the recent findings of Manuelidis and Borden (19) who reported on the localization of specific chromosomal domains in interphase nuclei of neurons and glia using 3-D reconstructions of serial sections from in situ-hybridized human central nervous system (CNS) tissue. Using biotinylated probes to centromeric sequences (alphoid repeats) these investigators showed that distinct cell populations organize their centromeric repeats in similar ways in mouse and human CNS cells. If spatial organization of genes, transcription, RNA processing, and nuclear transport exists, this will have important consequences for gene regulation. The further characterization of higher order organization of functional regions within the cell nucleus will provide a powerful approach by which to study cellular function.

I thank James D. Watson for his support and encouragement throughout the course of this work. The excellent technical assistance of Michael Delannoy is greatly appreciated. The artistic skills and patience of Jim Duffy, who provided many drafts of Fig. 4 before I was satisfied, are greatly appreciated as are the photographic skills of Phil Renna. In addition, I thank Wai-Kit Chan, Greg Conway, Robert Franza, Adrian Krainer, Mike Mathews, Barbara McClintock, and Rich Roberts for helpful discussions. This study was supported by American Cancer Society Grant NP-619 and by National Cancer Institute Grant 5P30 CA45508-01.

1. Foe, V. E. \& Alberts, B. M. (1983) J. Cell Sci. 61, 31-70.

2. Foe, V. E. \& Alberts, B. M. (1985) J. Cell Biol. 100, 1623-1636.

3. Agard, D. \& Sedat, J. (1983) Nature (London) 302, 676-681.

4. Hochstrasser, M. \& Sedat, J. (1987) J. Cell Biol. 104, 1455-1470.

5. Mathog, D., Hochstrasser, M., Gruenbaum, Y., Saumweber, H. \& Sedat, J. (1984) Nature (London) 308, 414-421.

6. Rykowski, M. C., Parmelee, S. J., Agard, D. A. \& Sedat, J. W. (1988) Cell 54, 461-472.

7. Byers, B. \& Porter, K. R. (1964) Proc. Natl. Acad. Sci. USA 52, 1091-1099.

8. Dustin, P. (1978) Microtubules (Springer New York), pp. 88-114.

9. MacGregor, H. C. \& Stebbings, H. (1970) J. Cell Sci. 6, 431-449.

10. Mollenhauer, H. H. (1974) J. Cell Sci. 15, 89-98.

11. Sandoval, I. V., Bonifacino, J. S., Klausner, R. D., Henkart, M. \& Wehland, J. (1984) J. Cell Biol. 99, 1135-1185.

12. Busch, H. \& Smetana, K. (1970) The Nucleolus (Academic New York).

13. Lawrence, J. B. \& Singer, R. H. (1986) Cell 45, 407-415.

14. Spector, D. L. \& Smith, H. C. (1986) Exp. Cell Res. 163, 87-94.

15. Lerner, E. A., Lerner, M. R., Janeway, C. A. \& Steitz, J. A. (1981) Proc. Natl. Acad. Sci. USA 78, 2737-2741.

16. Salpeter, M. M. \& Bachmann, L. (1964) J. Cell Biol. 22, 469-477.

17. Birnstiel, M. L. (1988) Structure and Function of Major and Minor Small Nuclear Ribonucleoprotein Particles (Springer, New York).

18. Krainer, A. R. \& Maniatis, T. (1988) in Transcription and Splicing, eds. Hames, B. D. \& Glover, D. M. (IRL, Washington, DC), pp. 131-206.

19. Manuelidis, L. \& Borden J. (1988) Chromosoma 96, 397-410.

20. Spector, D. L. (1984) Biol. Cell 51, 109-112.

21. Reuter, R., Appel, B., Bringmann, P., Rinke, J. \& Luhrmann, R. (1984) Exp. Cell Res. 154, 548-560.

22. Spector, D. L., Watt, R. A. \& Sullivan, N. F. (1987) Oncogene 1, 5-12.

23. Frenster, J. H. (1974) in The Cell Nucleus, ed. Busch, H. (Academic, New York), Vol. 1, pp. 565-580.

24. Beyer, A. L., Miller, O. L., Jr., \& McKnight, S. L. (1980) Cell 20, 75-84.

25. Fakan, S., Puvion, E. \& Spohr, G. (1976) Exp. Cell Res. 99, 155-164.

26. Zieve, G. \& Penman, S. (1976) Cell 8, 19-31.

27. Vasiliev, J. M. (1987) J. Cell Sci. Suppl. 8, 1-18.

28. Spector, D. L., Schrier, W. H. \& Busch, H. (1983) Biol. Cell 49, 1-10.

29. Smith, H. C., Ochs, R. L., Fernandez, E. A. \& Spector, D. L. (1986) Mol. Cell. Biochem. 70, 151-168.

30. Lawrence, J. B., Singer, R. H. \& Marselle, L. M. (1989) Cell 57, 493-502.

31. Dambaugh, T., Hennessy, K., Fennewald, S. \& Kieff, E. (1986) in The Epstein-Barr Virus: Recent Advances, eds. Epstein, M. A. \& Achong, B. G. (Wiley, New York), pp. 14-45.

32. Beyer, A. L. \& Osheim, Y. N. (1988) Genes Dev. 2, 754-765.

33. Webb, T. E., Schumm, D. E. \& Palayoor, T. (1981) in The Cell Nucleus, ed. Busch, H. (Academic, New York), Vol. 9, pp. 199-241.

34. Blobel, G. (1985) Proc. Natl. Acad. Sci. USA 82, 8527-8529. 